\title{
Altered fibrin clot properties in advanced lung cancer: impact of chemotherapy
}

\author{
Grzegorz Królczyk ${ }^{1,2 \#}$, Michał Ząbczyk ${ }^{3 \#}$, Grzegorz Czyżewicz ${ }^{1}$, Krzysztof Plens ${ }^{4}$, Shannon Prior ${ }^{5}$, \\ Saulius Butenas ${ }^{5}$, Anetta Undas ${ }^{3,6}$ \\ ${ }^{1}$ Oncology Ward, John Paul II Hospital, Cracow, Poland; ${ }^{2}$ Faculty of Medicine and Health Sciences, Andrzej Frycz Modrzewski Krakow University, \\ Cracow, Poland; ${ }^{3}$ Institute of Cardiology, Jagiellonian University Medical College and John Paul II Hospital, Cracow, Poland; ${ }^{4}$ KCRI, Cracow, \\ Poland; ${ }^{5}$ Department of Biochemistry, University of Vermont, Burlington, VT, USA; ${ }^{6}$ Faculty of Medicine and Health Sciences, Jan Kochanowski \\ University, Kielce, Poland \\ Contributions: (I) Conception and design: A Undas, G Królczyk; (II) Administrative support: A Undas, G Czyżewicz; (III) Provision of study materials \\ or patients: A Undas, G Królczyk, G Czyżewicz; (IV) Collection and assembly of data: A Undas, M Ząbczyk; (V) Data analysis and interpretation: M \\ Ząbczyk, S Prior, S Butenas; (VI) Manuscript writing: All authors; (VII) Final approval of manuscript: All authors. \\ \#These authors contributed equally to this work. \\ Correspondence to: Anetta Undas, MD, PhD. Institute of Cardiology, Jagiellonian University Medical College, 80 Pradnicka Str., 31-202 Kraków, \\ Poland. Email: mmundas@cyf-kr.edu.pl.
}

Background: Faster formation of dense and poorly lyzable fibrin networks have been reported in patients at risk of thromboembolism, including cancer patients. We sought to investigate whether chemotherapy affects plasma fibrin clot properties and their determinants in lung cancer patients.

Methods: In this observational study we enrolled 83 consecutive patients with advanced inoperable lung cancer. Plasma fibrin clot permeability $\left(\mathrm{K}_{\mathrm{s}}\right)$, turbidimetric analysis of clot formation, clot lysis time (CLT), microparticle-associated tissue factor (MP-TF) activity, and thrombin generation parameters were investigated at enrolment and 3-4 months after standard chemotherapy.

Results: Lung cancer patients after 4 (range, 4-5) cycles of chemotherapy had 35.6\% higher D-dimer, $22.1 \%$ lower MP-TF activity, and unaltered fibrinogen compared with baseline. Chemotherapy resulted also in $7.5 \%$ increased $\mathrm{K}_{\mathrm{s}}, 8.6 \%$ prolonged lag phase, and $5.4 \%$ shortened CLT, while thrombin generation was unchanged. Chemotherapy-related differences in clot structure were confirmed by scanning electron microscopy images. Fibrin clot properties after chemotherapy did not differ among histological types of lung cancer, cancer stages or chemotherapy regimens. Interestingly, never smoking $(n=13,16 \%)$ was associated with looser post-treatment fibrin structure as reflected by $12.3 \%$ higher $\mathrm{K}_{\mathrm{s}}$. Multiple linear regression showed that more advanced cancer stage, higher peak thrombin generation, and higher white blood cell count determined post-treatment change in $\mathrm{K}_{\mathrm{s}}$, while active smoking was associated with change in CLT.

Conclusions: Three-month chemotherapy in lung cancer patients improves clot properties despite unaffected thrombin generation, suggesting that anticancer treatment might quickly produce antithrombotic actions.

Keywords: Chemotherapy; clot lysis time (CLT); fibrin clot; lung cancer; thrombin generation

Submitted Aug 09, 2018. Accepted for publication Oct 17, 2018.

doi: $10.21037 /$ jtd.2018.11.19

View this article at: http://dx.doi.org/10.21037/jtd.2018.11.19 


\section{Introduction}

Lung cancer is the leading cause of cancer death among both men and women (1), however available treatment, including palliative strategies, significantly prolongs the life of patients with advanced lung cancer (2). Despite the introduction of new therapies such as chemotherapy and immunotherapy that in some cases make cancer a chronic disease, lung cancer remains the most prevalent fatal tumour (3). Approximately 3\% of lung cancer patients develop venous thromboembolism (VTE) within 2 years since diagnosis (4) with a year cumulative incidence of $10.2 \%$ in patients with small cell lung carcinoma (SCLC) (5) and about $22 \%$ in those with non-small cell lung carcinoma (NSCLC), which accounts for more than $80 \%$ lung cancers $(6,7)$. A hypercoagulable state in lung cancer involves tissue factor (TF), microparticles, anticoagulant and antifibrinolytic systems, platelets, and many other factors (8). The lung cancer chemotherapy is associated with three-fold increased risk for VTE (9). An imbalance between coagulation activation and fibrinolytic potential might contribute to thromboembolic complications during chemotherapy. Shortening of clotting time, increase in antithrombin levels and thrombin-antithrombin complexes, followed by decreased plasma D-dimer, plasmin- $\alpha_{2}$-antiplasmin complex and fibrinogen levels have been observed in lung cancer patients during the first and the second cycle of standard anticancer therapy with a large variability of these parameters up to 3 weeks after treatment (10). Recent study has demonstrated that activated and apoptotic platelets, together with plateletderived microparticles contribute to hypercoagulable state in patients with NSCLC after chemotherapy and can be closely related to thromboembolic complications during anticancer treatment (11).

Growing evidence indicates that the formation of clots composed of compact fibrin networks resistant to fibrinolysis, which is largely determined by environmental factors (12), predisposes to VTE (13). Cigarette smoking, a common risk factor for lung cancer, also negatively alters fibrin clot properties (14). Data on fibrin clot characteristics in cancer patients, however, are scarce. Less permeable fibrin clots relatively resistant to lysis have been observed in gastrointestinal cancer and multiple myeloma $(15,16)$. Rheometry of blood clots in lung cancer patients has shown the formation of pathological clot microstructure with changes of physical properties, including higher fractal dimension in patients with extensive lung cancer compared with the localized disease (17).

To our knowledge, comprehensive analysis of the plasma clot formation, permeability, and lysis in patients with advanced lung cancer during chemotherapy has not been published so far. Therefore, we investigated plasma fibrin clot features and their determinants in lung cancer patients before and after chemotherapy.

\section{Methods}

\section{Study population}

From May to September 2014 in a prospective study we evaluated 83 white consecutive patients with advanced histologically or cytologically confirmed lung cancer who were recruited at the Department of Oncology, the John Paul II Hospital, Cracow, Poland. Patients were categorized into subjects with SCLC and those with NSCLC that included three main subtypes: adenocarcinoma, squamous cell cancer, and not otherwise specified (NOS) carcinoma, involving cases other than adenocarcinoma, squamous cell, large cell carcinoma or mixed/other histology.

The American Joint Committee on Cancer (AJCC) stages were determined according to the AJCC 7 th edition staging system, using available clinical data. Initially all patients met all the following eligibility criteria for chemotherapy: an Eastern Cooperative Oncology Group (ECOG) performance status of 0 or 1 ; adequate organ function (leukocytes $\geq 3,000 / \mu \mathrm{L}$ and $\leq 12,000 / \mu \mathrm{L}$; neutrophils $\geq 1,500 / \mu \mathrm{L}$; platelets $\geq 100,000 / \mu \mathrm{L}$; haemoglobin $\geq 9.0 \mathrm{~g} / \mathrm{dL}$; total bilirubin $\leq 1.5 \mathrm{mg} / \mathrm{dL}$; aspartate aminotransferase and alanine aminotransferase $\leq 2.5 \times$ upper limit of normal; serum creatinine $\leq 1.5 \mathrm{mg} / \mathrm{dL}$ or creatinine clearance $\geq 60 \mathrm{~mL} / \mathrm{min} ; \mathrm{PaO}_{2} \geq 60 \mathrm{mmHg}$ ).

The exclusion criteria were: any active infections, glomerular filtration rate $<60 \mathrm{~mL} / \mathrm{min}$, hypo- or hyperthyroidism, any acute vascular events, and current anticoagulant therapy. Patients in whom prophylaxis with low-molecular-weight heparins (LMWH) was administered, were eligible.

Heart failure (HF) was defined as the presence of relevant symptoms and signs and left ventricular ejection fraction $\leq 45 \%$. Arterial hypertension was diagnosed based on a history of hypertension (blood pressure $>140 / 90 \mathrm{mmHg}$ ) or preadmission antihypertensive treatment. Diabetes mellitus was defined as fasting glucose $\geq 7.0 \mathrm{mM}$ on two separate occasions or use of insulin or oral hypoglycaemic 
agents. Coronary artery disease (CAD) was defined as hospitalization for angina, prior documented myocardial infarction or coronary revascularization. Ischemic stroke was diagnosed based on the World Health Organization criteria. Chronic obstructive pulmonary disease was defined by the presence of irreversible expiratory airflow limitation measured by spirometry. Current smoker was defined as a subject who has smoked 100 cigarettes and currently declares the use of one or more cigarettes per day, while former smoker as a person who has smoked at least 100 cigarettes in a lifetime, but currently (at least within the last month) does not smoke.

All patients commenced standard chemotherapy according to the histopathological type of cancer and co-morbidities (18). Patients on chemotherapy underwent standard chest computed tomography scans at the 9th or 12th week since its onset and were assessed according to the response evaluation criteria in solid tumours (RECIST 1.1) (19). The recommended thromboprophylaxis in high-risk patients was administered (20).

The Local Ethical Committee in Krakow approved the study (31/KBL/OIL/2013) and participants provided informed consent in accordance with the Declaration of Helsinki.

\section{Laboratory investigations}

Fasting blood samples were drawn from antecubital vein using minimal stasis. Blood samples were drawn at the time of enrolment before starting chemotherapy and before the third or fourth cycle of chemotherapy. Patients who received prophylactic LMWH were drawn at least 12 hours since the injection. Complete blood count, glucose and creatinine were assayed by routine laboratory techniques. Fibrinogen was determined using the Clauss method. Plasma D-dimer was measured with the Innovance D-dimer assay (Siemens, Marburg, Germany). Immunoenzymatic assay was used to determine the TF-bearing microparticles (MP-TF) activity (Hyphen BioMed, Neuville sur Oise, France).

\section{Fibrin clot properties}

In citrated plasma (vol/vol, 9:1 of 3.2\% sodium citrate), the following variables describing a plasma clot formation, structure and lyzability were determined in duplicate by technicians blinded to the origin of the samples (intra-assay and inter-assay coefficients of variation, $5 \%$ to $7 \%$ ).

\section{Clot permeability}

Permeation of plasma fibrin clots was determined as described (21). Briefly, $20 \mathrm{mM}$ calcium chloride and $1 \mathrm{U} / \mathrm{mL}$ human thrombin (Sigma-Aldrich, St. Louis, MO, USA) were added to citrated plasma. Tubes containing the clots were connected to a reservoir of a Tris-buffered saline (TBS; $0.01 \mathrm{M}$ Tris, $0.1 \mathrm{M} \mathrm{NaCl}, \mathrm{pH} 7.4$ ) and its volume flowing through the gels was measured. A permeation coefficient $\left(\mathrm{K}_{\mathrm{s}}\right)$, which indicates the pore size, was calculated from the equation: $K_{s}=Q x L x \eta / t x A x \Delta p$, where $Q$ is the flow rate in time $t, L$ is the length of a fibrin gel, $\eta$ is the viscosity of liquid (in poise), $\mathrm{t}$ is percolating time, $\mathrm{A}$ is the cross-sectional area (in $\mathrm{cm}^{2}$ ) and $\Delta \mathrm{p}$ is a differential pressure (in dyne $/ \mathrm{cm}^{2}$ ).

After $\mathrm{K}_{\mathrm{s}}$ measurement clots ( $\left.\mathrm{n}=6\right)$ were fixed using $2.5 \%$ glutaraldehyde, then removed from tubes, washed with distilled water, dehydrated in graded water-ethanol solutions, dried by the critical point procedure and sputter coated with gold. Samples were scanned in six different areas (microscope JEOL JCM-6000; JEOL Ltd., Tokyo, Japan).

\section{Turbidity measurements}

Plasma citrated samples were mixed 2:1 with a TBS containing $0.6 \mathrm{U} / \mathrm{mL}$ human thrombin (Sigma-Aldrich) and $50 \mathrm{mM} \mathrm{CaCl}_{2}$ to initiate polymerization. Absorbance was read at $405 \mathrm{~nm}$ with a Perkin-Elmer Lambda 4B spectrophotometer (Molecular Devices). The lag phase of the turbidity curve, which reflects the time required for initial protofibril formation and maximum absorbance at the plateau phase $(\Delta \mathrm{Abs})$, indicating the number of protofibrils per fiber, were recorded (21).

\section{Clot lysis assay}

Clot lysis time (CLT) was measured as described previously (21). Briefly, to $75 \mu \mathrm{L}$ of citrated plasma we added TF (dilution 105 times; Innovin, Dade Behring, Deerfield, IL, USA), $\mathrm{CaCl}_{2}$ (final concentration, $17 \mathrm{mM}$ ), tissue plasminogen activator (tPA, final concentration, $30 \mathrm{U} / \mathrm{mL}$; Boehringer Ingelheim, Ingelheim, Germany) and phospholipid vesicles (22) (final concentration, $10 \mathrm{mM}$ ). HEPES buffer was added to make a total volume of $150 \mu \mathrm{L}$.

\section{Thrombin generation assay}

To assess the thrombin generation profiles, we used the assay previously described $(23,24)$. Briefly, corn trypsin inhibitor was added to citrated plasma $(0.1 \mathrm{mg} / \mathrm{mL}$, final concentration) and $80 \mu \mathrm{L}$ of the sample was mixed with relipidated TF ( $5 \mathrm{pM}$, final concentration). Twenty $\mu \mathrm{L}$ of a 
$2.5 \mathrm{mM}$ Z-GGR-AMC/90 $\mathrm{mM} \mathrm{CaCl} \mathrm{Cl}_{2}$ solution in HEPES was added. Twenty $\mu \mathrm{L}$ of a $120 \mu \mathrm{M}$ phospholipid vesicles solution in HEPES was then added to plasma samples to achieve a final concentration of $20 \mu \mathrm{M}$, thus initiating thrombin generation. Hydrolysis of the AMC substrate (at 370/460 nm) was followed over a 3,600 s period. Changes in fluorescence were converted to thrombin concentration using a calibration curve built by sequential dilutions of human thrombin.

\section{Statistical analysis}

The study was powered to have a $90 \%$ chance of detecting a $10 \%$ difference in CLT using a $\mathrm{P}$ value of 0.01 , based on the previous study (25). In order to demonstrate such a difference, or a greater one, 32 patients or more were required in each group. In turn, to demonstrate such a difference, or a greater one, in $\mathrm{K}_{\mathrm{s}}$ using a $\mathrm{P}$ value of 0.05 at least 31 patients were required in each group (22).

Variables were presented as mean and standard deviation, median and interquartile range or otherwise stated. The normality of distribution was checked using ShapiroWilk test. The Spearman's rho correlation coefficient was computed to measure the relationship between continuous variables. For testing association for categorical variables, the Fischer's Exact test was used. The $t$-test was used for means comparison, whereas the non-parametric U MannWhitney or Kruskal-Wallis tests were used for comparison of non-normally distributed variables. For paired data the paired Student's $t$-test or the Wilcoxon signed-rank tests were used as appropriate.

$\Delta \mathrm{K}_{\mathrm{s}}$ and $\triangle \mathrm{CLT}$ were calculated as a difference in $\mathrm{K}_{\mathrm{s}}$ values after 3 -month chemotherapy and baseline, and the difference in the CLT values between baseline and after 3-month chemotherapy, respectively. Higher $\Delta \mathrm{K}_{\mathrm{s}}$ and $\triangle$ CLT denote improved clot permeability and lyzability, respectively. Determinants of $\Delta \mathrm{K}_{\mathrm{s}}$ and $\Delta$ CLT were established in a multiple linear regression model, built by a forward stepwise selection procedure, and verified by $\mathrm{F}$ Snedecor's statistics, with $\mathrm{F}>1$. The $\mathrm{R}^{2}$ was used as a measure of the variance. The models were adjusted for fibrinogen. All statistical analyses were performed with $\mathrm{JMP}^{\circledR}$, version 12.2.0 (SAS Institute INC., Cary, NC, USA).

\section{Results}

\section{Patient characteristics}

Baseline characteristics of lung cancer patients before and after therapy are summarized in Table 1. At enrolment 37 (44.6\%) patients were diagnosed with the SCLC and 46 (55.4\%) with the NSCLC. Metastatic lung cancer was diagnosed in $53(63.9 \%)$ patients, while in $30(36.1 \%)$ locally advanced inoperable lung cancer was recognized. The study group included 37 patients with limited disease (LD) and 46 patients with extended disease (ED).

Patients with NSCLC before chemotherapy had higher peak thrombin $\{181$ [108-247] vs. 135 [97-148] nM, $\mathrm{P}=0.043\}$ and $\mathrm{D}$-dimer $\{528[351-1,085]$ vs. 324 [254-691] $\mathrm{ng} / \mathrm{mL}, \mathrm{P}=0.017\}$ levels than those with SCLC, while patients with stage IV/ED were characterized by higher D-dimer $\{638$ [396-1,174] vs. 326 [254-495] ng/mL, $\mathrm{P}<0.0001\}$ and MP-TF activity $[1.74(1.24-2.40)$ vs. 1.30 (0.89-1.90) $\mathrm{pg} / \mathrm{mL}, \mathrm{P}=0.032]$ compared to those with stage III/LD.

\section{Impact of chemotherapy}

Among 83 patients who completed at least 4 [median, 5 (range, 4-5)] cycles of chemotherapy, 12 (14.5\%) subjects were treated with cisplatin/vinorelbine, one subject $(1.2 \%)$ with cisplatin/gemcitabine, 5 (6\%) subjects with carboplatin/vinorelbine, 13 (15.7\%) with paclitaxel/ carboplatin, 10 (12\%) with cisplatin/pemetrexed, $1(1.2 \%)$ with vinorelbine in monotherapy, 23 (27.7\%) with cisplatin/etoposide and 17 (20.5\%) with carboplatin/ etoposide. In one $(1.2 \%)$ patient the tyrosine kinase inhibitor was administered. Among lung cancer patients, $54(65 \%)$ individuals responded to treatment, 21 (25\%) had disease stabilization and $8(10 \%)$ had cancer progression. Interestingly, CLT assessed before chemotherapy was significantly associated with the clinical response to treatment and was prolonged in patients with the disease progression and shortened in those with the response to chemotherapy $(\mathrm{P}$ value for ANOVA $=0.038$; Figure 1$)$. No symptomatic thromboembolic events were observed.

Three months after the onset of chemotherapy we found $17.5 \%$ lower red blood cell count, $28.6 \%$ lower white blood cell count, $15.5 \%$ lower haemoglobin, along with $35.6 \%$ higher D-dimer and 22.1\% lower MP-TF activity, while fibrinogen was unaltered. Interestingly, we observed $7.5 \%$ increased $\mathrm{K}_{\mathrm{s}}, 8.6 \%$ prolonged lag phase, $5.4 \%$ shortened CLT and unchanged thrombin generation parameters (Table 1). As reflected by the SEM analysis, along with the clot permeability, patients after chemotherapy had more porous fibrin structure (Figure 2). Patients with NSCLC compared to those with SCLC had higher glucose levels, 
Table 1 Laboratory tests and fibrin clot variables in lung cancer patients before and after chemotherapy

\begin{tabular}{|c|c|c|c|}
\hline Variable & Lung cancer patients $(\mathrm{n}=83)$ & $\begin{array}{l}\text { Patients after 3-month chemotherapy } \\
\qquad(\mathrm{n}=83)\end{array}$ & $P$ value \\
\hline Age, years & $63.9 \pm 7.0$ & - & - \\
\hline Body mass index, $\mathrm{kg} / \mathrm{m}^{2}$ & $25.3 \pm 4.6$ & - & - \\
\hline CAD, n (\%) & $14(16.9)$ & - & - \\
\hline Arterial hypertension, n (\%) & $41(49.4)$ & - & - \\
\hline Diabetes, n (\%) & $9(10.8)$ & - & - \\
\hline Aspirin, n (\%) & $16(19.3)$ & - & - \\
\hline RBC, $10^{6} / \mu \mathrm{L}$ & $4.51 \pm 0.46$ & $3.73 \pm 0.52$ & $<0.001$ \\
\hline WBC, $10^{3} / \mu \mathrm{L}$ & $9.0[7.2-11.4]$ & $6.5[5.1-9.0]$ & $<0.001$ \\
\hline Platelets, $10^{3} / \mu \mathrm{L}$ & 313 [256-366] & 324 [221-394] & 0.92 \\
\hline Haemoglobin, g/dL & $12.9 \pm 1.5$ & $10.9 \pm 1.4$ & $<0.001$ \\
\hline Glucose, mM & $5.3[4.9-6.0]$ & $5.5[5.1-6.1]$ & 0.40 \\
\hline Creatinine, $\mu \mathrm{M}$ & 74 [62-84] & 77 [67-90] & 0.075 \\
\hline$\Delta$ Abs $(405 \mathrm{~nm})$ & $0.85[0.80-0.90]$ & $0.84[0.78-0.88]$ & $0.80^{*}$ \\
\hline Clot lysis time, min & $98.0 \pm 16.8$ & $92.7 \pm 15.2$ & $0.031^{*}$ \\
\hline Lag time, s & $1,177[1,028-1,483]$ & $1,119[917-1,301]$ & 0.18 \\
\hline Peak thrombin generated, nM & 144 [98-223] & 136 [99-205] & 0.22 \\
\hline TTPeak, s & $2,139[1,854-2,585]$ & $2,063[1,740-2,386]$ & 0.97 \\
\hline ETP, nM·s & $94,346 \pm 37,237$ & $91,744 \pm 35,278$ & 0.095 \\
\hline
\end{tabular}

*, adjusted for fibrinogen. Data are given as mean $\pm \mathrm{SD}, \mathrm{n}(\%)$, or median [interquartile range]. COPD, chronic obstructive lung disease; CAD, coronary artery disease; HF, heart failure; ACEl, angiotensin-converting enzyme inhibitors; RBC, red blood cells; WBC, white blood cells; MP-TF, tissue factor-bearing microparticles procoagulant activity; $\mathrm{K}_{\mathrm{s}}$, fibrin clot permeability; $\triangle \mathrm{Abs}(405 \mathrm{~nm})$, maximum absorbance of fibrin gel at $405 \mathrm{~nm}$; TTPeak, time to thrombin peak; ETP, endogenous thrombin potential. 


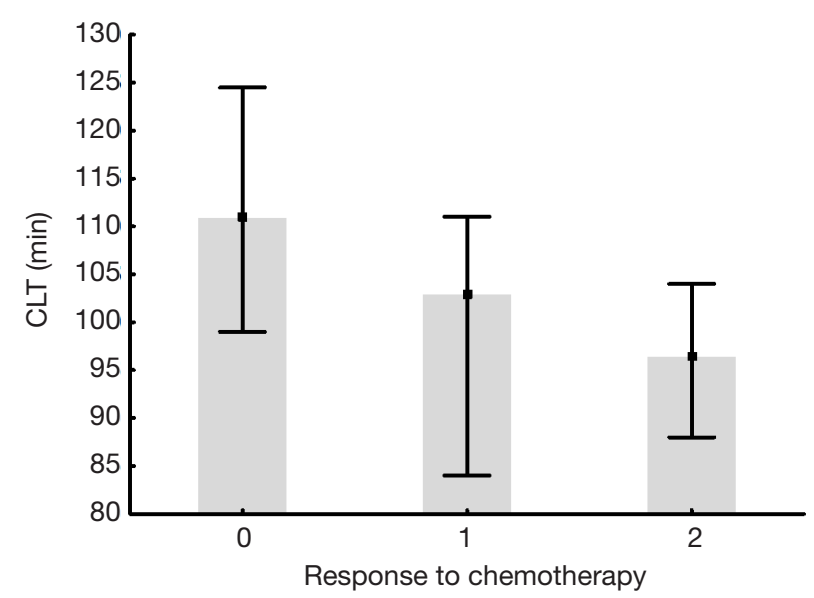

Figure 1 Associations between CLT and response to chemotherapy in lung cancer patients. 0, denotes cancer progression; 1 , disease stabilization; 2, any response to treatment. Data are given as median (interquartile range). CLT, clot lysis time.

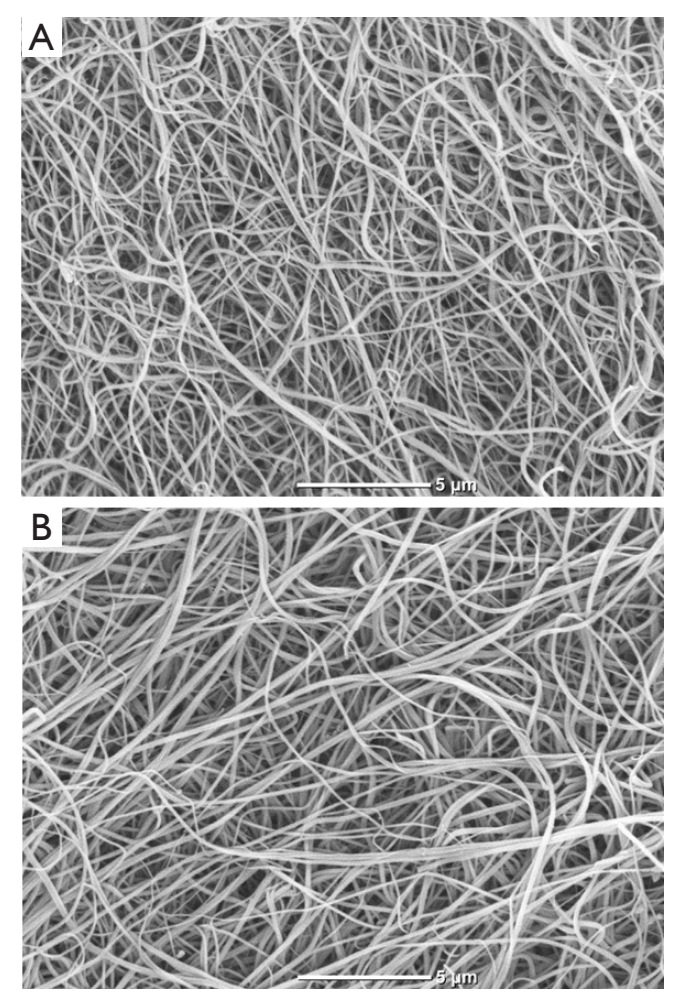

Figure 2 Representative scanning electron microscopy (SEM) images of plasma fibrin networks in a representative lung cancer patient before (A) and after chemotherapy (B). Magnification, $5,000 \times$; fibrinogen concentration, $3 \mathrm{~g} / \mathrm{L} . \mathrm{K}_{\mathrm{s}}=6.3 \times 10^{-9} \mathrm{~cm}^{2}$ before chemotherapy (A) and $\mathrm{K}_{\mathrm{s}}=7.4 \times 10^{-9} \mathrm{~cm}^{2}$ after anticancer treatment (B). peak thrombin generated, and ETP, while subjects with stage ED/IV had higher D-dimer levels and peak thrombin generated than those with LD/III (Table 2). None of the analysed variables differed significantly between patients receiving various chemotherapy combinations (data not shown). Fibrin clot properties after chemotherapy were similar in patients with different histological types of lung cancer or cancer stages (Table 2). Notably, $\mathrm{K}_{\mathrm{s}}$, but not other fibrin or thrombin generation variables, was higher after chemotherapy in never smoking patients $(n=13,16 \%)$ compared with current and former smokers [8.1 (7.5-8.2) vs. $\left.7.1(6.7-7.5) \times 10^{-9} \mathrm{~cm}^{2}, \mathrm{P}=0.046\right]$. Post-treatment $\mathrm{K}_{\mathrm{s}}$ was associated with the pack-years $(\mathrm{r}=-0.29, \mathrm{P}=0.016)$ and with MP-TF activity $(\mathrm{r}=-0.23, \mathrm{P}=0.037)$. Peak thrombin generated correlated with $\mathrm{K}_{\mathrm{s}}$, WBC count, and D-dimer $(\mathrm{r}=-0.31, \mathrm{P}=0.0091 ; \mathrm{r}=0.46, \mathrm{P}<0.001$ and $\mathrm{r}=-0.29, \mathrm{P}=0.015$, respectively), while ETP was associated with $\mathrm{WBC}$ only $(\mathrm{r}=0.31, \mathrm{P}=0.0071)$.

The multiple linear regression model showed that stage $\mathrm{ED} / \mathrm{IV}\left(\mathrm{B}=-0.31 ; 95 \% \mathrm{CI}:-0.57\right.$ to $-0.056 ; \mathrm{R}^{2}=33$, $\mathrm{P}<0.0001$ ), peak thrombin generated (B per $10 \mathrm{nM}=-0.030$; 95\% CI: -0.059 to $-0.001 ; \mathrm{R}^{2}=39, \mathrm{P}<0.0001$ ), and white blood cell count (B per $10^{3} / \mu \mathrm{L}=-0.082 ; 95 \%$ CI: -0.15 to $\left.-0.011 ; \mathrm{R}^{2}=32, \mathrm{P}<0.0001\right)$ determined $\Delta \mathrm{K}_{\mathrm{s}}$, while active smoking determined $\triangle \mathrm{CLT}$ ( $\mathrm{B}=-4.95 \mathrm{~min}$; 95\% CI: -9.64 to $\left.-0.27 \mathrm{~min} ; \mathrm{R}^{2}=0.37, \mathrm{P}<0.0001\right)$.

\section{Discussion}

Recently, we have demonstrated that the prothrombotic plasma clot phenotype, including faster formation of fibrin clots, reduced fibrin network porosity and impaired clot lyzability, characterizes patients with advanced lung cancer, including locally advanced inoperable disease and disseminated disease (Abstract No. PB 912 at the XXVIth Congress of the ISTH). The current study is the first to show that a 3-month chemotherapy in lung cancer patients has a favourable effect on fibrin clot properties, including slower formation of less compact fibrin networks, despite unaltered persistently elevated thrombin generation. Our study provides new insights into the mechanisms of a hypercoagulable state in lung cancer patients during chemotherapy (9), suggesting the presence of additional favourable fibrin-related changes, in part determined by the disease stage, thrombin generation potential, and smoking habit.

A slight but significant improvement in fibrin clot properties assessed after 3 months of standard chemotherapy 


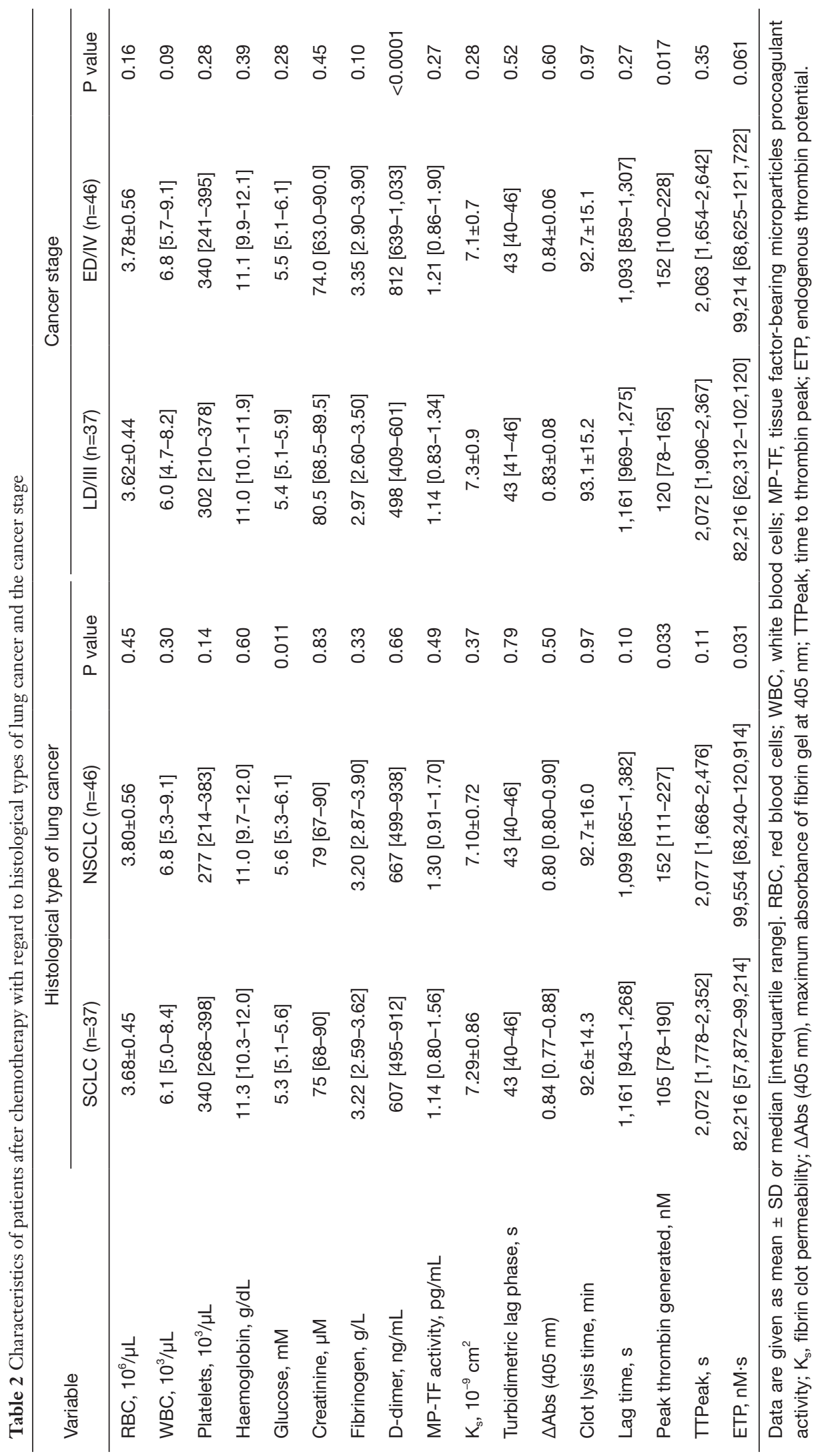


in advanced lung cancer patients was a surprising finding, since typically anticancer therapy is associated with a hypercoagulable state reflected by increased fibrinogen and D-dimer levels $(9,26)$. In our previous study performed in multiple myeloma patients induction therapy led to higher $\mathrm{K}_{\mathrm{s}}$ and shortened CLT associated with a reduction in peak thrombin generated (27). In advanced cancer patients, no such associations were observed. Thrombin and TF have been postulated to represent key mediators of cancerrelated thrombosis in general (28). Reitter et al. (29) have shown that peak thrombin generated decreased in lung cancer patients at different disease stages over time, while D-dimer levels increased during chemotherapy. The authors examined, however, less than one third of patients with the advanced disease (29). We observed no significant differences regarding thrombin generation in the current study, which might suggest that 3 months of chemotherapy in patients with advanced inoperable lung cancer has a negligible impact on thrombin formation, but MP-TF activity was reduced by $22 \%$ after treatment. Increased circulating levels of MPs have been shown in lung cancer patients compared with healthy controls (30). Highly elevated MP-TF activity was also found in patients with metastatic pancreatic cancer, with its moderate inverse association with time to fibrin clot formation (31). Few studies have evaluated MP-TF activity in lung cancer patients (17). We found a weak association of MP-TF with $\mathrm{K}_{\mathrm{s}}$ after treatment, which suggests an additional harmful effect of elevated MP-TF activity and its contribution to the formation of denser, more prothrombotic fibrin networks. This observation is novel and might have a large impact in other non-cancer clinical settings. The current study provides additional evidence that a coagulation potential of TF-bearing MPs might contribute to the multifactorial hypercoagulability in lung cancer patients.

We confirmed the strong impact of smoking on plasma clot structure in lung cancer patients, which highlights a major prothrombotic effect of this factor, potent enough to be observed despite a highly prothrombotic state in cancer. Cigarette smoking, in particular in heavy smokers, increases thrombotic risk via multiple mechanisms, including hyperfibrinogenaemia or enhanced oxidative stress leading to impaired fibrin permeability and lysis (32). We demonstrated that the active or former smoking was associated with about $12 \%$ lower $\mathrm{K}_{\mathrm{s}}$ after chemotherapy, which indicates that negative effects of smoking on clot properties are still detectable during chemotherapy. Of note, these effects of smoking were not related to differences in fibrinogen concentrations or thrombin generation. Our observation regarding smoking underscores the value of recommendations to quit smoking in cancer and non-cancer patients, also in order to positively impact prothrombotic tendencies. We showed that no smoking can determine a more favourable post-treatment clot feature. It has also been reported that never smokers with NSCLC had higher response rates to chemotherapy, lower rates of progressive disease and longer survival than current and former smokers (33). Based on the current evidence, it might be speculated that less prothrombotic fibrin clot phenotype might also be related to a better outcome during anticancer therapy.

Recently, it has been suggested that platelets might play an important role in hypercoagulable state development after chemotherapy (11). Platelets behind blood coagulation are involved in angiogenesis and cancer growth by secretion of multiple growth factors and chemoattractants, including platelet factor 4 (34,35). In vitro experiments on platelets treated with cisplatin showed that anticancer drugs can lead to increased production of intrinsic activated factor $\mathrm{X}$, followed by elevated thrombin generation and fibrin formation (11). Thrombin formation and fibrin generation were increased 1 day after chemotherapy and returned to the baseline (day 0) after one week in a model using platelet-free plasma mixed with platelets isolated from blood of lung cancer patients (11). How important is the platelet activation in the development of thromboembolic complications in cancer patients in the light of our data showing favourable changes in fibrin properties after chemotherapy needs further investigation.

This study has several limitations. First, the sample size was limited. The study was, however, adequately powered to detect intergroup differences in clot variables, although the subgroup analyses should be interpreted with caution. Second, the present cohort included various histological types of lung cancer and treatment with a few regimens, however such group is representative for this disease at its advanced stage. Third, an assessment of platelet activation was beyond the scope of this study.

In conclusion, advanced lung cancer unfavourably alters plasma clot properties, including faster formation of more compact clots displaying impaired lyzability, but chemotherapy improves these properties. Clinical relevance of the current findings remains to be explored.

\section{Acknowledgements}

We thank Alexander Olson for his technical assistance. 
Funding: This work was supported by the Polish National Science Centre (grant number UMO-2013/09/B/ NZ5/00254 to A Undas) and by the National Institutes of Health (grant number UM1 HL120877 TACTIC to S Butenas).

\section{Footnote}

Conflicts of Interest: The authors have no conflicts of interest to declare.

Ethical Statement: The Local Ethical Committee in Krakow approved the study (31/KBL/OIL/2013) and participants provided informed consent in accordance with the Declaration of Helsinki.

\section{References}

1. Ridge CA, McErlean AM, Ginsberg MS. Epidemiology of lung cancer. Semin Intervent Radiol 2013;30:93-8.

2. Bansal P, Osman D, Gan GN, et al. Recent Advances in Targetable Therapeutics in Metastatic Non-Squamous NSCLC. Front Oncol 2016;6:112.

3. Mao Y, Yang D, He J, et al. Epidemiology of Lung Cancer. Surg Oncol Clin N Am 2016;25:439-45.

4. Chew HK, Davies AM, Wun T, et al. The incidence of venous thromboembolism among patients with primary lung cancer. J Thromb Haemost 2008;6:601-8.

5. Lee YG, Lee E, Kim I, et al. Cisplatin-Based Chemotherapy Is a Strong Risk Factor for Thromboembolic Events in Small-Cell Lung Cancer. Cancer Res Treat 2015;47:670-5.

6. Numico G, Garrone O, Dongiovanni V, et al. Prospective evaluation of major vascular events in patients with nonsmall cell lung carcinoma treated with cisplatin and gemcitabine. Cancer 2005;103:994-9.

7. Li R, Hermann G, Baldini E, et al. Advanced nodal stage predicts venous thromboembolism in patients with locally advanced non-small cell lung cancer. Lung Cancer 2016;96:41-7.

8. Walker AJ, Baldwin DR, Card TR, et al. Risk of venous thromboembolism in people with lung cancer: A cohort study using linked UK healthcare data. Br J Cancer 2016;115:115-21.

9. Huang H, Korn JR, Mallick R, et al. Incidence of venous thromboembolism among chemotherapy-treated patients with lung cancer and its association with mortality: a retrospective database study. J Thromb Thrombolysis
2012;34:446-56.

10. Gabazza EC, Taguchi O, Yamakami T, et al. Alteration of coagulation and fibrinolysis systems after multidrug anticancer therapy for lung cancer. Eur J Cancer 1994;30A:1276-81.

11. Ma R, Bi Y, Kou J, et al. Enhanced procoagulant activity of platelets after chemotherapy in non-small cell lung cancer. Cancer Biol Ther 2017;18:627-34.

12. Undas A, Ariëns RA. Fibrin clot structure and function: a role in the pathophysiology of arterial and venous thromboembolic diseases. Arterioscler Thromb Vasc Biol 2011;31:e88-99.

13. Ząbczyk M, Undas A. Plasma fibrin clot structure and thromboembolism: clinical implications. Pol Arch Intern Med 2017;127:873-81.

14. Barua RS, Sy F, Srikanth S, et al. Acute cigarette smoke exposure reduces clot lysis - association between altered fibrin architecture and the response to t-PA. Thromb Res 2010;126:426-30.

15. Gronostaj K, Richter P, Nowak W, et al. Altered plasma fibrin clot properties in patients with digestive tract cancers: links with the increased thrombin generation. Thromb Res 2013;131:262-7.

16. Undas A, Zubkiewicz-Usnarska L, Helbig G, et al. Altered plasma fibrin clot properties and fibrinolysis in patients with multiple myeloma. Eur J Clin Invest 2014;44:557-66.

17. Davies NA, Harrison NK, Morris RH, et al. Fractal dimension (df) as a new structural biomarker of clot microstructure in different stages of lung cancer. Thromb Haemost 2015;114:1251-9.

18. European Society for Medical Oncology. ESMO Clinical Practice Guidelines: Lung and Chest Tumours. Available online: http://www.esmo.org/Guidelines/Lung-andChest-Tumours [Accessed August 3, 2017].

19. Eisenhauer EA, Therasse P, Bogaerts J, et al. New response evaluation criteria in solid tumours: revised RECIST guideline (version 1.1). Eur J Cancer 2009;45:228-47.

20. Krasiński Z, Krasińska B, Dzieciuchowicz $€$, et al. Heparins in cancer-associated venous thrombosis. Pol Arch Med Wewn 2016;126:419-29.

21. Undas A, Zawilska K, Ciesla-Dul M, et al. Altered fibrin clot structure/function in patients with idiopathic venous thromboembolism and in their relatives. Blood 2009;114:4272-8.

22. Lisman T, de Groot PG, Meijers JC, et al. Reduced plasma fibrinolytic potential is a risk factor for venous thrombosis. Blood 2005;105:1102-5.

23. Mann KG, Whelihan MF, Butenas S, et al. Citrate 
anticoagulation and the dynamics of thrombin generation. J Thromb Haemost 2007;5:2055-61.

24. Hemker HC, Giesen P, Al Dieri R, et al. Calibrated automated thrombin generation measurement in clotting plasma. Pathophysiol Haemost Thromb 2003;33:4-15.

25. Meltzer ME, Lisman T, de Groot PG, et al. Venous thrombosis risk associated with plasma hypofibrinolysis is explained by elevated plasma levels of TAFI and PAI-1. Blood 2010;116:113-21.

26. Zhu LR, Li J, Chen P, et al. Clinical significance of plasma fibrinogen and D-dimer in predicting the chemotherapy efficacy and prognosis for small cell lung cancer patients. Clin Transl Oncol 2016;18:178-88.

27. Undas A, Zubkiewicz-Usnarska L, Helbig G, et al. Induction therapy alters plasma fibrin clot properties in multiple myeloma patients: association with thromboembolic complications. Blood Coagul Fibrinolysis 2015;26:621-7.

28. Rickles FR, Patierno S, Fernandez PM. Tissue factor, thrombin, and cancer. Chest 2003; 124:58S-68S.

29. Reitter EM, Kaider A, Ay C, et al. Longitudinal analysis of hemostasis biomarkers in cancer patients during antitumor treatment. J Thromb Haemost 2016;14:294-305.

30. Tseng CC, Wang CC, Chang HC, et al. Levels of circulating microparticles in lung cancer patients and possible prognostic value. Dis Markers 2013;35:301-10.

31. Thaler J, Koder S, Kornek G, et al. Microparticleassociated tissue factor activity in patients with metastatic pancreatic cancer and its effect on fibrin clot formation. Transl Res 2014;163:145-50.

32. Undas A, Topór-Madry R, Tracz W, et al. Effect of cigarette smoking on plasma fibrin clot permeability and susceptibility to lysis. Thromb Haemost 2009;102:1289-91.

33. Tsao AS, Liu D, Lee JJ, et al. Smoking affects treatment outcome in patients with advanced nonsmall cell lung cancer. Cancer 2006;106:2428-36.

34. Gay LJ, Felding-Habermann B. Contribution of platelets to tumour metastasis. Nat Rev Cancer 2011;11:123-34.

35. Pucci F, Rickelt S, Newton AP, et al. PF4 Promotes Platelet Production and Lung Cancer Growth. Cell Rep 2016;17:1764-72.
Cite this article as: Królczyk G, Ząbczyk M, Czyżewicz G, Plens K, Prior S, Butenas S, Undas A. Altered fibrin clot properties in advanced lung cancer: impact of chemotherapy. J Thorac Dis 2018;10(12):6863-6872. doi: 10.21037/ jtd.2018.11.19 